\title{
A SPECTRAL RADIUS ESTIMATE AND ENTROPY OF HYPERCUBES
}

\author{
WILLIAM GELLER, BRUCE KITCHENS, MICHA£ MISIUREWICZ, AND MICHA£ RAMS
}

\begin{abstract}
We consider a problem in Mathematical Biology that leads to a question in Graph Theory, which can be solved using an old but not widely known upper estimate of the spectral radius of a nonnegative matrix. We provide a new proof of this estimate.
\end{abstract}

\section{INTRODUCTION}

One of the methods of studying systems arising in biology is to replace all values that can be attained by any given variable by just two values: below and above some threshold (see, e.g., $[3,4,11,13]$ ). Then the evolution in time will be represented by a path on a corresponding graph: each variable can switch from one state to the other, depending on the values of all the remaining variables. If there are $k$ variables, since generically only one variable can switch at any time, the graph that we should consider is the 1-skeleton of the $k$-dimensional hypercube. Since for each variable, the values of all other variables determine how this one will change, each edge of the graph has its direction. This way we get a digraph.

The complexity of our system (how many paths are possible?) can be measured by the entropy of the graph. Wilds, Kaufmann and Glass [14] asked the question: what is the maximal entropy of the $k$-dimensional hypercube over all possible assignments of directions of edges? We learned about this question from Leon Glass. In [14] the authors conjecture, based on numerical experiments, that this maximal entropy is $\log \frac{k}{2}$ if $k$ is even and $\log \frac{\sqrt{k^{2}-1}}{2}$ if $k$ is odd. Here we prove that indeed this is the case.

Note that if instead of a hypercube we consider a complete graph, a similar question has been studied extensively, see [2] (the digraphs one gets are called tournaments).

The entropy of a digraph is equal to the logarithm of the spectral radius of the transition (adjacency) matrix of this graph (or 0 if this spectral radius is 1 or less). Thus, we need to estimate the spectral radius of this matrix. This problem plays an important role in several branches of mathematics; apart from Linear Algebra and Graph Theory, we can list Dynamical Systems, Probability Theory and applications of mathematics. There are many different estimates, see, e.g., [8]. In particular, the following simple estimate is well known. Let $M$ be an $n \times n$ nonnegative matrix with

Date: November 8, 2011.

2000 Mathematics Subject Classification. 15A18, 15A48, 05C20, 37B10, 92B05.

Key words and phrases. Nonnegative matrices, spectral radius, topological entropy, entropy of graphs, biological dynamics.

M.R. was partially supported by MNiSWN grant N201 607640 (Poland). 
row sums $R_{i}$, column sums $C_{i}$, and spectral radius $\lambda$. Then

$$
\begin{aligned}
& \min \left\{R_{i}: i=1, \ldots, n\right\} \leq \lambda \leq \max \left\{R_{i}: i=1, \ldots, n\right\}, \\
& \min \left\{C_{i}: i=1, \ldots, n\right\} \leq \lambda \leq \max \left\{C_{i}: i=1, \ldots, n\right\} .
\end{aligned}
$$

However, there are cases when we would like to get a better estimate, knowing the row and column sums of $M$. Such an estimate,

$$
\lambda \leq \max \left\{\sqrt{R_{i} C_{i}}: i=1, \ldots, n\right\},
$$

has been obtained by E. W. Barankin in [1] and rediscovered by J. Kwapisz in [6]. It has been strengthened by A. Ostrowski in [10] to the following one:

$$
\lambda \leq \max \left\{R_{i}^{p} C_{i}^{1-p}: 1 \leq i \leq n\right\}
$$

for every $p \in(0,1)$. Note that we get only upper estimates; the lower ones similar to (1.1) do not hold.

Inequalities (1.2) and (1.3) remain surprisingly unknown. Usually they do not appear even in the books with many different estimates of the spectral radii of nonnegative matrices. The survey article [2] of R. A. Brualdi mentions the result of Kwapisz, but does not [1] or [10]. However, it cites a paper [5] of L. Yu. Kolotilina, which cites the paper of Ostrowski (and his result), which cites the paper of Barankin. This way, after we rediscovered (1.2) and (1.3), we learned that we were 66 and 60 years late.

Nevertheless, our proof of (1.3) ((1.2) is a special case of (1.3) for $p=1 / 2)$ is quite different, and simpler, than the original proof of Ostrowski. We present it here, as well as the explanation of the ideas from Dynamical Systems that led to this proof. We should note that Kwapisz's proof also uses ideas from Dynamical Systems, but different ones than used by us.

The paper is organized as follows. In Section 2 we give a simple algebraic proof of (1.3). In Section 3 we explain briefly how we got this proof using ideas from Dynamical Systems. This section is purely explanatory; it is not necessary to read it in order to understand the rest of the paper. In Section 4 we translate our results to the language of Graph Theory and derive some interesting consequences. In Section 5

we apply the results of the preceding section to the problem in Mathematical Biology that we mentioned. Finally, in Section 6 we give two examples. The first one shows that we cannot count on lower estimates of the spectral radius using estimates similar to (1.3). The second one shows that (1.3) is stronger than (1.2).

\section{Spectral Radius estimate}

In this section we give a simple proof of the theorem of Ostrowski.

Theorem 2.1. Let $M$ be an $n \times n$ nonnegative matrix with row sums $R_{i}$, column sums $C_{i}$, and spectral radius $\lambda$. Then for every $p \in(0,1)$

$$
\lambda \leq \max \left\{R_{i}^{p} C_{i}^{1-p}: 1 \leq i \leq n\right\} .
$$

Proof. Let $M=\left(m_{i j}\right)$ and assume first that $M$ is positive. There are positive eigenvectors of $M$ corresponding to the eigenvalue $\lambda$ : a left one $\left(\ell_{i}\right)$ and a right one $\left(r_{i}\right)$. 
That is,

$$
\sum_{i=1}^{n} \ell_{i} m_{i j}=\lambda \ell_{j}, \quad \sum_{j=1}^{n} m_{i j} r_{j}=\lambda r_{i}
$$

We choose those eigenvectors in such a way that

$$
\sum_{i=1}^{n} \ell_{i} r_{i}=1
$$

By (2.2) and (2.3) we get

$$
\begin{aligned}
& \sum_{i, j=1}^{n} \frac{\ell_{i} m_{i j} r_{j}}{\lambda} \log \frac{\lambda r_{i}}{r_{j}} \\
&=\sum_{i, j=1}^{n} \frac{\ell_{i} m_{i j} r_{j}}{\lambda} \log \lambda+\sum_{i, j=1}^{n} \frac{\ell_{i} m_{i j} r_{j}}{\lambda} \log r_{i}-\sum_{i, j=1}^{n} \frac{\ell_{i} m_{i j} r_{j}}{\lambda} \log r_{j} \\
&=\sum_{j=1}^{n} \ell_{j} r_{j} \log \lambda+\sum_{i=1}^{n} \ell_{i} r_{i} \log r_{i}-\sum_{j=1}^{n} \ell_{j} r_{j} \log r_{j}=\log \lambda
\end{aligned}
$$

By (2.2), for each $i$ we have

$$
\sum_{j=1}^{n} \frac{m_{i j} r_{j}}{\lambda r_{i}}=1
$$

Thus, since the logarithmic function is concave, we get by Jensen's inequality

$$
\sum_{j=1}^{n} \frac{m_{i j} r_{j}}{\lambda r_{i}} \log \frac{\lambda r_{i}}{r_{j}} \leq \log \sum_{j=1}^{n} \frac{m_{i j} r_{j}}{\lambda r_{i}} \cdot \frac{\lambda r_{i}}{r_{j}}=\log R_{i}
$$

Now, from (2.4) and (2.5) we get

$$
\log \lambda=\sum_{i=1}^{n} \ell_{i} r_{i} \sum_{j=1}^{n} \frac{m_{i j} r_{j}}{\lambda r_{i}} \log \frac{\lambda r_{i}}{r_{j}} \leq \sum_{i=1}^{n} \ell_{i} r_{i} \log R_{i} .
$$

When we replace the matrix $M$ by its transpose, $\ell_{i}$ 's and $r_{i}$ 's will switch and $R_{i}$ 's will become $C_{i}$ 's. Thus,

$$
\log \lambda \leq \sum_{i=1}^{n} \ell_{i} r_{i} \log C_{i}
$$

By (2.6) and (2.7), we get for every $p \in(0,1)$

$$
\log \lambda \leq p \sum_{i=1}^{n} \ell_{i} r_{i} \log R_{i}+(1-p) \sum_{i=1}^{n} \ell_{i} r_{i} \log C_{i}=\sum_{i=1}^{n} \ell_{i} r_{i}\left(p \log R_{i}+(1-p) \log C_{i}\right)
$$

In view of (2.3), this gives us

$$
\log \lambda \leq \max \left\{p \log R_{i}+(1-p) \log C_{i}: 1 \leq i \leq n\right\}
$$

so (2.1) follows. 
Now, if $M$ is just nonnegative instead of positive, for every $\varepsilon>0$ we consider a positive matrix $M_{\varepsilon}=\left(m_{i j}+\varepsilon\right)$ with row sums $R_{\varepsilon, i}$, column sums $C_{\varepsilon, i}$ and spectral radius $\lambda_{\varepsilon}$. By what we proved, we have

$$
\lambda_{\varepsilon} \leq \max \left\{R_{\varepsilon, i}^{p} C_{\varepsilon, i}^{1-p}: 1 \leq i \leq n\right\} .
$$

Clearly, $\lambda \leq \lambda_{\varepsilon}$. Moreover, for every $i$

$$
\lim _{\varepsilon \rightarrow 0} R_{\varepsilon, i}^{p} C_{\varepsilon, i}^{1-p}=R_{i}^{p} C_{i}^{1-p}
$$

Thus, we get (2.1) by taking the limit as $\varepsilon \rightarrow 0$ in (2.10).

\section{Ideas From Dynamical Systems}

Now we explain the source of the proof from the preceding section. This is not essential for the proof, so we will omit definitions. The reader who needs them can find them in many textbooks in Dynamical Systems (a standard one is [12]).

For a positive $n \times n$ nonnegative matrix with row sums $R_{i}$, column sums $C_{i}$, and spectral radius $\lambda$, we consider the two-sided full shift on $n$ symbols $\sigma: \Sigma \rightarrow \Sigma$. Denote the $i$-th 1 -cylinder by $[i]$ and the $(i, j)$-th 2 -cylinder by $[i j]$. Let $f: \Sigma \rightarrow \mathbb{R}$ be the function constant on 2-cylinders that takes value $m_{i j}$ on $[i j]$. Then the topological pressure $P(\sigma, \log f)$ is equal to $\log \lambda$ and there is a probability measure $\mu$ on $\Sigma$, invariant for $\sigma$, which is the equilibrium state for $(\sigma, \log f)$. Moreover, the system $(\Sigma, \sigma, \mu)$ is a stationary Markov chain. If $p_{i}=\mu([i])$ and the transition probabilities are $p_{i j}$, then

$$
P(\sigma, \log f)=-\sum_{i, j=1}^{n} p_{i} p_{i j} \log p_{i j}+\sum_{i, j=1}^{n} p_{i} p_{i j} \log m_{i j}
$$

Using an inequality that is standard in the proof of the Variational Principle (see, e.g., formula (6) of [9]), one gets

$$
P(\sigma, \log f) \leq \sum_{i=1}^{n} p_{i} \log R_{i}
$$

Now instead of $\sigma$ we consider $\sigma^{-1}$. This corresponds to replacing the matrix $M$ by its transpose. They have the same spectral radii. Moreover, the pressure and metric entropy are preserved by taking the inverse of the map, and the system $\left(\Sigma, \sigma^{-1}, \mu\right)$ is also a stationary Markov chain with the same measures of cylinders $[i]$ and transition probabilities $p_{j i} p_{j} / p_{i}$. However, the rows of $M^{T}$ are the columns of $M$, so we get now

$$
P(\sigma, \log f) \leq \sum_{i=1}^{n} p_{i} \log C_{i}
$$

In this way we get (2.6) and (2.7), and the proof follows as in the preceding section.

\section{ENTROPY OF GRAPHS}

A special case of nonnegative matrices and their spectral radii, when the matrix has only entries 0 and 1, can be interpreted in terms of Dynamical Systems or Graph Theory.

Thus, if $M=\left(m_{i j}\right)$ is a $0-1$ matrix, we can consider the space

$$
\Sigma_{M}=\left\{\left(x_{k}\right)_{k=-\infty}^{\infty}: \forall_{k} x_{k} \in\{1, \ldots, n\} \text { and } m_{x_{k} x_{k+1}}=1\right\}
$$


with the topology of a subset of $\{1, \ldots, n\}^{\mathbb{Z}}$ with the product topology (in each $\{1, \ldots, n\}$ we take the discrete topology). Further, $\sigma_{M}: \Sigma_{M} \rightarrow \Sigma_{M}$ is the shift to the left, that is,

$$
\sigma_{M}\left(\left(x_{k}\right)\right)=\left(y_{k}\right), \text { where } y_{k}=x_{k+1} .
$$

The system $\left(\Sigma_{M}, \sigma_{M}\right)$ is the subshift of finite type with transition matrix $M$. The logarithm of the spectral radius of $M$ is equal to the exponential growth rate of the number of cylinders of length $k$ (the sets of sequences with prescribed terms at coordinates $0,1, \ldots, k-1$ ), and is the topological entropy of $\sigma_{M}$.

Alternatively, we can think of a directed graph (digraph) $G$ with $n$ vertices, in which there is an arrow from the $i$-th vertex to the $j$-th one if and only if $m_{i j}=1$. Then the logarithm of the spectral radius of $M$ (we will call it the entropy of $G$ and denote it $h(G)$ ) is the exponential growth rate of the number of paths of length $k$ in $G$.

The number of arrows beginning in a given vertex $v$ is the outdegree of $v$; the number of arrows ending at $v$ is the indegree of $v$. Note that the outdegree of the $i$-th vertex of $G$ is the $i$-th row sum of $M$, and the indegree of the $i$-th vertex of $G$ is the $i$-th column sum of $M$. Thus, we can restate Theorem 2.1 as follows.

Theorem 4.1. Let $G$ be a digraph with $n$ vertices. Let the outdegree of the $i$-th vertex be $d_{i}^{+}$and its indegree $d_{i}^{-}$. Then for every $p \in[0,1]$

$$
h(G) \leq \max \left\{p \log d_{i}^{+}+(1-p) \log d_{i}^{-}: 1 \leq i \leq n\right\},
$$

(unless the right-hand side is $-\infty$ ).

Here we were able to add 0 and 1 as possible values of $p$ to incorporate the trivial upper estimates of (1.1).

In fact, using (1.1), we get the following simple lemma.

Lemma 4.2. If the outdegree of each vertex of $G$ (or the indegree of each vertex of $G)$ is $k$ then $h(G)=\log k$.

Let us define the capacity of a vertex of $G$ as the geometric mean of its outdegree and indegree. Then from Theorem 4.1 we get immediately a version of the theorem of Barankin.

Corollary 4.3. The entropy of a digraph is not larger than the maximum of the logarithms of the capacities of its vertices.

Now we consider the following problem. For a digraph $G$ let us denote by $|G|$ an undirected graph that is obtained from $G$ by replacing each arrow by an edge. We assume that in $G$ if there is an arrow from a vertex $v$ to a vertex $u$ then there is no arrow from $u$ to $v$, so the graph $|G|$ is simple (i.e. has no multiple edges between a pair of vertices or self loops at a vertex).

Problem 4.4. For a given simple undirected graph $H$, find

$$
h_{\text {dir }}(H):=\max \{h(G):|G|=H\} .
$$

Lemma 4.2 and Corollary 4.3 provide a quick solution in the case when $H$ is a $k$-regular graph (the degree of each vertex of $H$ is $k$ ) with $k$ even. 
Theorem 4.5. If $H$ is a $k$-regular simple undirected graph and $k$ is even, then

$$
h_{\text {dir }}(H)=\log \frac{k}{2}
$$

Proof. If $G$ is any digraph with $|G|=H$, then the capacity of every vertex of $G$ is the geometric mean of two nonnegative integers whose sum is $k$. By the inequality between the geometric and arithmetic means, we see that this capacity is not larger than $k / 2$, so by Corollary $4.3, h(G) \leq \log (k / 2)$. Thus, $h_{d i r}(H) \leq \log (k / 2)$.

On the other hand, by the theorem stated by Euler in 1736 and proved by Hierholzer in 1873, since the degree of each vertex is even, there exists an Eulerian circuit (a closed path that follows each edge of $H$ exactly once). When we follow it and orient edges accordingly, we get a digraph $G$ with the indegree and outdegree of each vertex equal to $k / 2$. Now, by Lemma $4.2, h(G)=\log (k / 2)$. Thus, $h_{d i r}(H) \geq \log (k / 2)$.

In order to get a similar result for $k$ odd, we have to add an assumption that $H$ is bipartite. This means that the set of vertices of $H$ can be divided into two subsets, such that there are no edges joining vertices from the same subset.

Theorem 4.6. If $H$ is a k-regular simple bipartite undirected graph and $k$ is odd, then

$$
h_{d i r}(H)=\log \frac{\sqrt{k^{2}-1}}{2} .
$$

Proof. If $G$ is any digraph with $|G|=H$, then the capacity of every vertex of $G$ is the geometric mean of two nonnegative integers whose sum is $k$. Therefore, this capacity is not larger than $((k+1) / 2) \cdot((k-1) / 2)=\left(k^{2}-1\right) / 4$, so by Corollary 4.3 , $h(G) \leq \log \left(\sqrt{k^{2}-1} / 2\right)$. This proves that $h_{\text {dir }}(H) \leq \log \left(\sqrt{k^{2}-1} / 2\right)$.

Since the graph $H$ is bipartite, there exists a partition of the set of vertices of $H$ into two sets, $X$ and $Y$, such that every edge of $H$ joins a vertex from $X$ with a vertex from $Y$. Since the degree of every vertex of $H$ is $k$, by Theorem 2 of [7] (a simple consequence of the Marriage Lemma), there is a one-to-one function $\varphi: X \rightarrow Y$, such that every $x \in X$ is joined by an edge with $\varphi(x)$. Similarly, there is a one-to-one function $\psi: Y \rightarrow X$, such that every $y \in Y$ is joined by an edge with $\psi(y)$. In particular, the cardinalities of $X$ and $Y$ are equal, so $\varphi$ is also onto. When we erase all edges from $x$ to $\varphi(x)$, we get a graph $H^{\prime}$ which is $(k-1)$-regular. Thus, as in the proof of Theorem 4.5, we can orient the edges of $H^{\prime}$ in such a way that each vertex has outdegree and indegree $(k-1) / 2$. Now we orient the removed edges of $H$ to go from $X$ to $Y$, and in such a way we get a digraph $G$ with $|G|=H$ such that all vertices from $X$ have outdegree $(k+1) / 2$ and indegree $(k-1) / 2$, while all vertices from $Y$ have outdegree $(k-1) / 2$ and indegree $(k+1) / 2$.

Let $M$ be the transition matrix of $G$. Then $M^{2}$ has row sums

$$
\frac{k+1}{2} \cdot \frac{k-1}{2}=\frac{k^{2}-1}{4}
$$

so by (1.1), its spectral radius is $\left(k^{2}-1\right) / 4$. Thus, the spectral radius of $M$ is $\sqrt{k^{2}-1} / 2$. This proves that $h_{d i r}(H) \geq \log \left(\sqrt{k^{2}-1} / 2\right)$. 


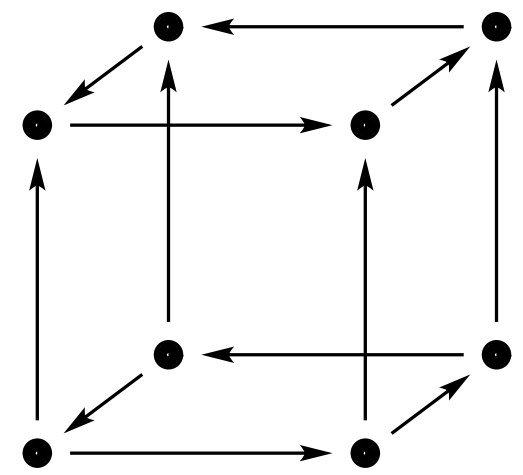

FiguRE 1. Entropy zero digraph with positive capacities.

\section{Application to Biological Systems}

Now we consider the question mentioned in the Introduction: what is the maximal entropy of the $k$-dimensional hypercube over all possible assignments of directions of edges? This is our Problem 4.4 in the case when $H$ is the 1-skeleton of the $k$ dimensional hypercube. The results of the preceding section give immediately the answer.

Theorem 5.1. If $H$ is the 1-skeleton of the $k$-dimensional hypercube then

(a) if $k$ is even, then $h_{d i r}(H)=\log \frac{k}{2}$;

(b) if $k$ is odd, then $h_{\text {dir }}(H)=\log \frac{\sqrt{k^{2}-1}}{2}$.

Proof. The graph $H$ is a $k$-regular simple undirected graph, so in view of Theorems 4.5 and 4.6, the only thing we have to prove is that if $k$ is odd then $H$ is bipartite. To show this, we embed the hypercube into $\mathbb{R}^{k}$ in a natural way, with vertices at $\{0,1\}^{k}$. Then we divide the set of vertices into two sets, according whether the sum of coordinates of the vertex is even or odd. The endpoints of each edge differ exactly at one coordinate, so they belong to different sets of the partition. This completes the proof.

\section{EXAMPLES}

In this section we provide two examples. The first one shows that in Theorem 2.1 we do not get a lower estimate of the spectral radius by the minimum of $R_{i}^{p} C_{i}^{1-p}$, even for the matrices obtained from hypercubes, as in Section 5.

Consider the graph from Figure 1. Four lower vertices have outdegree 2 and indegree 1 , while four upper vertices have outdegree 1 and indegree 2 . Thus, if $p \in(0,1)$, the values of $R_{i}^{p} C_{i}^{1-p}$ are $2^{p}$ and $2^{1-p}$; all of them are positive. Nevertheless, all paths either stay in the bottom and follow a period 4 circuit, or go to the top and there follow the period 4 circuit. Therefore, their number grows linearly with the length of the path, so the entropy of the graph is 0 . 
The second example shows that the estimate from Theorem 2.1 is stronger than its special case with $p=1 / 2$. Let $M$ be the following matrix:

$$
\left(\begin{array}{llll}
0 & 2 & 1 & 1 \\
0 & 0 & 2 & 0 \\
1 & 0 & 0 & 1 \\
0 & 0 & 1 & 0
\end{array}\right)
$$

The row sums are $4,2,2,1$, and the column sums $1,2,4,2$. The numbers $R_{i}^{p} C_{i}^{1-p}$ with $p=1 / 2$ are $2,2,2 \sqrt{2}, \sqrt{2}$, so their maximum is

$$
2 \sqrt{2} \approx 2.828427125
$$

On the other hand, the numbers $R_{i}^{p} C_{i}^{1-p}$ with $p=2 / 3$ are $2 \sqrt[3]{2}, 2,2 \sqrt[3]{2}, \sqrt[3]{2}$, so their maximum is

$$
2 \sqrt[3]{2} \approx 2.519842100
$$

Of course, this is just an upper estimate; the spectral radius of $M$ is equal to the largest root of the equation $x^{3}-2 x-5=0$, that is, approximately 2.094551482. A similar but more complicated example can be constructed on a hypercube.

\section{REFERENCES}

[1] E. W. Barankin, Bounds for the characteristic roots of a matrix, Bull. Amer. Math. Soc. 51 (1945), 767-770.

[2] R. A. Brualdi, Spectra of digraphs, Linear Algebra Appl. 432 (2010), 2181-2213.

[3] L. Glass and J. S. Pasternack, Stable oscillations in mathematical models of biological control systems, J. Math. Biology 6 (1978), 207-223.

[4] L. Glass and H. T. Siegelmann, Logical and symbolic analysis of robust biological dynamics, Current Opinion in Genetics \& Development 20 (2010), 644-649.

[5] L. Yu. Kolotilina, Bounds and inequalities for the Perron root of a nonnegative matrix (Russian), Zap. Nauchn. Sem. POMI 284 (2002), 77-122 (English version: J. Math. Sci. 121 (2004), 24812507).

[6] J. Kwapisz, On the spectral radius of a directed graph, J. Graph Theory 23 (1996), 405-411.

[7] A. Lenard, An application of the Marriage Lemma, Mathematics Magazine 74 (2001), 234-238.

[8] H. Minc, "Nonnegative matrices", John Wiley \& Sons, New York, 1988.

[9] M. Misiurewicz, A short proof of the variational principle for a $\mathbb{Z}_{+}^{N}$ action on a compact space, Astérisque 40 (1976), 147-157.

[10] A. Ostrowski, Ueber das Nichtverschwinden einer Klasse von Determinanten und die Lokalisierung der charakteristischen Wurzeln von Matrizen (German), Compositio Math. 9 (1951), 209-226.

[11] T. J. Perkins, R. Wilds and L. Glass, Robust dynamics in minimal hybrid models of genetic networks, Phil. Trans. R. Soc. A 368 (2010), 4961-4975.

[12] P. Walters, "An introduction to ergodic theory", Springer-Verlag, New York-Berlin, 1982.

[13] R. Wilds and L. Glass, An atlas of robust, stable, high-dimensional limit cycles, International Journal of Bifurcation and Chaos 19 (2009), 4055-4096.

[14] R. Wilds, S. A. Kauffman and L. Glass, Evolution of complex dynamics, Chaos 18 (2008), 033109 . 
Department of Mathematical Sciences, iUPui, 402 N. Blackford Street, IndiANAPOLIS, IN 46202-3216, USA

E-mail address: wgeller@math.iupui.edu

Department of Mathematical Sciences, iUPui, 402 N. Blackford Street, IndiANAPOLIS, IN 46202-3216, USA

E-mail address: bkitchens@math.iupui.edu

Department of Mathematical Sciences, iUpUi, 402 N. Blackford Street, IndiANAPOLIS, IN 46202-3216, USA

E-mail address: mmisiure@math.iupui.edu

Institute of Mathematics of the Polish Academy of Sciences, Śniadeckich 8, 00-956 Warszawa, Poland

E-mail address: rams@impan.pl 